\title{
miR-483-5p promotes growth, invasion and self-renewal of gastric cancer stem cells by Wnt/ $\beta$-catenin signaling
}

\author{
KAI WU, LONGAN MA and JINXIANG ZHU \\ Department of General Surgery, The Third Affiliated Hospital (Shaanxi Provincial People's Hospital), \\ Medical College of Xi'an Jiaotong University, Xi'an, Shaanxi 710061, P.R. China
}

Received May 10, 2015; Accepted July 11, 2016

DOI: $10.3892 / \mathrm{mmr} .2016 .5603$

\begin{abstract}
Gastric carcinoma (GC) ranks as the second most common cause of cancer-associated mortality worldwide. Emerging evidence has suggested a potential novel therapeutic strategy based on the ability of cancer stem cells (CSCs) to trigger tumorigenesis. MicroRNAs (miRNAs) have previously been implicated in CSC formation and regulation of their functional characteristics. In the current study, a significant upregulation of miR-483-5p levels was demonstrated in spheroid body-forming cells $(\mathrm{P}<0.01)$ by reverse transcription-quantitative polymerase chain reaction, which were isolated from the MKN-45 gastric cancer cell line and possessed gastric CSC (GCSC) properties. An MTT assay demonstrated that overexpression of miR-483-5p by transfection with miR-483-5p mimics significantly increased cell proliferation and Annexin V-propidium iodide staining indicated the suppression of cell apoptosis, suggesting that miR-483-5p has an important function in GCSC growth. Notably, Transwell and sphere formation assays demonstrated that miR-483-5p elevation promoted GCSC invasion and cell self-renewal ability, respectively. Further western blotting assays demonstrated that miR-483-5p upregulation induced an increase in the protein expression levels of $\beta$-catenin and its downstream target molecules, including cyclin D1, Bcl-2 and matrix metalloproteinase 2, indicating that miR-483-5p activates $\mathrm{Wnt} / \beta$-catenin signaling. Inhibition of this pathway by $\beta$-catenin small interfering RNA transfection attenuated the miR-483-5p-induced effects on cell growth, invasion and self-renewal. These results demonstrate that miR-483-5p may act as an oncogene to promote the development of GC by regulating GCSC growth, invasion and self-renewal via the Wnt/ $\beta$-catenin signaling pathway. Thus, the present study
\end{abstract}

Correspondence to: Mr. Kai Wu, Department of General Surgery, The Third Affiliated Hospital (Shaanxi Provincial People's Hospital), Medical College of Xi'an Jiaotong University, 309 West Yantan Road, Xi'an, Shaanxi 710061, P.R. China

E-mail: kaiwurmyy@163.com

Key words: gastric cancer, cancer stem cell, microRNA-483-5p, cell growth, cell invasion, self-renewal suggests that miR-483-5p may be a promising therapeutic target against $\mathrm{GC}$.

\section{Introduction}

Gastric carcinoma (GC) is considered to be the fourth most common type of malignancy worldwide and ranks as the second leading cause of cancer-associated mortality, following lung cancer $(1,2)$. Despite advances made in the treatment of GC, including surgical resection, chemotherapy and radiation therapy, the results are often disappointing, with a high recurrence rate of $70 \%$ following successful resection (3). Furthermore, the survival of patients is poor with a $28 \% 5$-year overall survival rate. Thus, a deeper understanding of the molecular mechanisms involved in controlling the initiation and progression of $\mathrm{GC}$ is imperative.

Previous studies have increasingly focused on the cancer stem cell (CSC) theory and its crucial function in the development and progression of cancer (4-6). A growing body of evidence has confirmed that CSCs comprise a small proportion of cancer cells with the ability to initiate tumor development and are recognized as the 'heartbeat' of cancer. The existence of CSCs has been identified in several types of cancer, including breast cancer, stomach cancer and glioma (7-9). Accumulating evidence has demonstrated that CSCs are involved in tumor aggressiveness, chemoresistance and metastasis, which may explain the high frequency of carcinoma relapse (4). However, a greater understanding of the underlying mechanisms associated with CSCs may provide potential novel therapeutic strategies for cancer.

MicroRNAs (miRNAs) are a family of endogenous small non-coding RNA molecules 21-24 nucleotides in length, which can interact with the 3'-untranslated region (3'-UTR) of target-mRNAs to induce translational repression and gene silencing. The dysregulation of miRNAs has been shown to interfere with various biological functions, including cell proliferation, invasion, metastasis and differentiation $(10,11)$. Emerging evidence has demonstrated the abnormal expression of miRNAs in several types of cancer, and has indicated that miRNAs can function as oncogenes or tumor suppressors to participate in carcinogenesis and progression (12-14). Previous studies reported that miRNAs can regulate the function of CSCs $(15,16)$. One such miRNA, miR-483-5p was demonstrated to be dysregulated in several cancers and identified as 
a potential carcinoma biomarker in certain types of cancer, including hepatocellular carcinoma, multiple myeloma and adrenocortical tumors $(17,18)$. In lung adenocarcinoma, miR-483-5p can promote epithelial-mesenchymal transition and enhance invasive and metastatic properties (19). However, the function and the underlying molecular mechanisms of miR-483-5p in the development of GC remains unclear.

The present study analyzed the expression of miR-483-5p in gastric CSCs (GCSCs) derived from the MKN-45 human gastric cancer cell line. The effects of miR-483-5p on cell growth, invasion and self-renewal of GCSCs, and the underlying molecular mechanisms were investigated.

\section{Materials and methods}

Reagents. Unless otherwise stated, all reagents were obtained from Sigma-Aldrich (St. Louis, MO, USA). The primary rabbit monoclonal antibodies against human $\beta$-catenin (cat. no. ab32572), Bcl-2 (cat. no. ab32124) and cyclin D1 (cat. no. ab134175) were purchased from Abcam (Cambridge, MA, USA). The mouse monoclonal antibody against matrix metalloproteinase 2 (MMP-2; cat. no. sc-13594) and the rabbit polyclonal antibody Ki67 (cat. no. sc-15402) were purchased from Santa Cruz Biotechnology, Inc. (Dallas, TX, USA).

Culture of parental and spheroid body-forming cells. MKN-45 human gastric cancer cells were obtained from the American Type Culture Collection (Manassas, VA, USA). Cells were maintained in RPMI-1640 medium supplemented with $10 \%$ fetal bovine serum (FBS; Sigma-Aldrich) and 1\% penicillin-streptomycin sulfate (Sigma-Aldrich). All cells were cultured in a humidified atmosphere at $37^{\circ} \mathrm{C}$ with $5 \% \mathrm{CO}_{2}$. Following the attachment of cells, they were subsequently passaged upon reaching $70-80 \%$ confluence. To obtain the spheroid bodies, the parental cells (100 cells/well) were plated in a 96-well plate and incubated with serum-free RPMI-1640 medium containing $100 \mathrm{ng} / \mathrm{ml}$ epidermal growth factor (EGF; Amyjet Scientific, Wuhan, China), $20 \mathrm{ng} / \mathrm{ml}$ human fibroblast growth factor-2 (FGF-2; Amyjet Scientific), 2\% B-27 supplement (Gibco; Thermo Fisher Scientific, Inc., Waltham, MA, USA) and 1\% N-2 supplement (Gibco; Thermo Fisher Scientific, Inc.). After 2 weeks, the primary spheroid body formation was evaluated and quantified using an inverted microscope (IX70; Olympus Corporation, Tokyo, Japan). When reaching a size of 200-500 cells per spheroid body, they were then collected and dissociated by trypsinization for passaging, and further amplification was performed as previously described (20).

Identification of parental-derived GCSCs by flow cytometric analysis. For flow cytometry, $80 \%$ confluent cells were detached from culture plates using $0.25 \%$ trypsin. Following centrifugation at $800 \mathrm{x}$ g for $5 \mathrm{~min}$, cells were resuspended in Hanks' balanced salt solution (HBSS) containing $1 \mathrm{mM}$ 4-(2-hydroxyethyl)-1-piperazineethanesulfonic acid (Gibco; Thermo Fisher Scientific, Inc.) and 2\% FBS. Cells were then filtered using a 40- $\mu \mathrm{m}$ mesh filter (BD Biosciences, San Jose, CA, USA). To identify the induced GCSCs, cells were plated onto glass coverslips and fixed with $4 \%$ paraformaldehyde (Sigma-Aldrich). After rinsing with phosphate-buffered saline (PBS; Sigma-Aldrich), cells were permeabilized with
$0.1 \%$ Triton X-100 (Sigma-Aldrich) in PBS for $10 \mathrm{~min}$. Following incubation with $1 \%(\mathrm{w} / \mathrm{v})$ solution of bovine serum albumin (BSA; Sigma-Aldrich) in PBS for 30 min to block the non-specific binding. Subsequently, a mouse anti-human CD44-allophycocyanin monoclonal antibody (BD Biosciences; cat. no. 559942), a common specific marker for GCSCs, was added at a dilution of $1: 400$ at $37^{\circ} \mathrm{C}$ with $5 \% \mathrm{CO}_{2}$. All samples were stained with $2 \mathrm{ng} / \mathrm{ml}$ of 4',6-diamidino-2-phenylindole (Sigma-Aldrich) to label DNA. After $30 \mathrm{mins}$, cells were rinsed with HBSS, then the collected samples were analyzed by flow cytometry using a FACSAria flow cytometer (BD Biosciences). The results were analyzed using software FlowJo version 7.2.4 (Tree Star Inc., Ashland, OR).

Oligonucleotide transfection. To specifically induce miR-483-5p expression in GCSCs derived from MKN-45 cells, miR-483-5p mimics and scrambled control oligonucleotides were synthesized and obtained from Shanghai GenePharma Co., Ltd. (Shanghai, China). For transfection, cells were seeded into 96-well plates and transfected with the above oligonucleotides using Lipofectamine 2000 reagent (Invitrogen; Thermo Fisher Scientific, Inc.) according to the manufacturer's protocol. After $48 \mathrm{~h}$ transfection, cells were collected and reverse transcription-quantitative polymerase chain reaction (RT-qPCR) was performed to evaluate the transfection effectiveness.

Transfection of synthetic $\beta$-catenin small interfering (si)RNA. Scrambled siRNA and siRNA targeting human $\beta$-catenin fragments were purchased from Shanghai GenePharma Co., Ltd. For siRNA transfection, cells with elevated miR-483-5p and control cells were cultured in 24-well plates and then transfected with $2 \mu \mathrm{g} / \mathrm{ml} \beta$-catenin siRNA mixed with $5 \mu \mathrm{l}$ Lipofectamine RNAi MAX (Invitrogen; Thermo Fisher Scientific, Inc.). Following incubation at room temperature for $24 \mathrm{~h}$, cells were collected and the efficiency of the $\beta$-catenin siRNA transfection was confirmed by western blot analysis.

$R T-q P C R$ analysis. Total RNA was extracted from cultured cells using TRIzol reagent (Invitrogen; Thermo Fisher Scientific, Inc.). Then, first-strand cDNA was obtained by RT using ImProm II Reverse Transcriptase (Promega Corporation, Madison, WI, USA) with oligo-dT primers from $2 \mu \mathrm{g}$ RNA. The obtained cDNA was subsequently subjected to qPCR using an SYBR Premix Ex Taq II kit (Takara Biotechnology Co., Ltd., Dalian, China) in a $20 \mu \mathrm{l}$ reaction mixture following the manufacturer's instructions. The thermocycling conditions were as follows: Initiation at $95^{\circ} \mathrm{C}$ for $3 \mathrm{~min} ; 40$ cycles of $95^{\circ} \mathrm{C}$ for $15 \mathrm{sec}$ and $60^{\circ} \mathrm{C}$ for $35 \mathrm{sec}$. The reaction was conducted in an ABI 7900 system (Applied Biosystems; Thermo Fisher Scientific, Inc.) and the results were analyzed using SDS 2.3 software (Applied Biosystems; Thermo Fisher Scientific, Inc.). The specific primers for CD44, Oct4, Nanog, Sox2, CK14, CK18 and miR-483-5p were used as previously described $(20,21)$. The quantity of PCR product was normalized with respect to $\mathrm{U} 6$ or $\beta$-actin as the endogenous controls. The results were quantified using the $2^{-\Delta \Delta \mathrm{Cq}}$ method (22).

Proliferation assays. Cell proliferation was measured using 3-(4,5-dimethylthiazol-2-yl)-2,5-diphenyltetrazolium bromide 

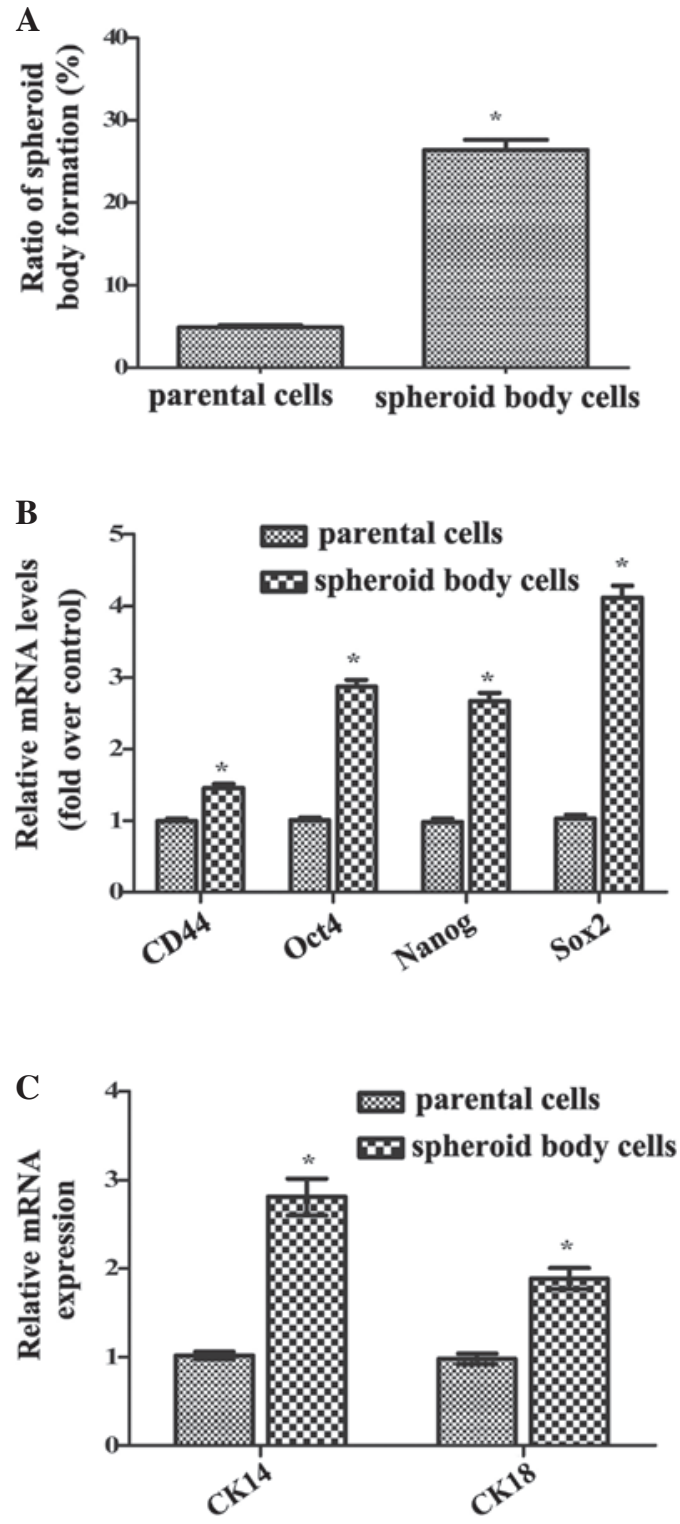

Figure 1. Isolation and identification of spheroid bodies derived from MKN-45 cells. (A) The FACS-sorted CD44 $4^{+}$MKN-45 cells were cultured in a low-attachment plate with serum-free media. After 2 weeks, the ratio of sphere formation was detected and compared with parental cells. (B) The mRNA levels of (B) CD44, Oct4, Nanog and Sox2, and (C) CK14 and CK18 were determined in spheroid-body cells and parental cells by reverse transcription-quantitative polymerase chain reaction. Values are presented as the mean \pm standard deviation. ${ }^{*} \mathrm{P}<0.05$ vs. the parental group. $\mathrm{CD}$, cluster of differentiation; CK, cytokeratin.

(MTT) assays. Briefly, cells were plated in 96-well plates and treated as described. Following washing with PBS, $20 \mu 1$ of $5 \mathrm{mg} / \mathrm{ml}$ MTT (Sigma-Aldrich) was added into each corresponding test well and incubated for $5 \mathrm{~h}$. Following removal of the supernatant, $200 \mu \mathrm{l}$ dimethyl sulfoxide was introduced to dissolve the formazan. The absorbance at $570 \mathrm{~nm}$ was detected to analyze cell viability using a micro-ELISA reader (Bio-Rad Laboratories, Inc., Hercules, CA, USA).

Apoptosis analysis. The rate of apoptosis was assessed by Annexin V-propidium iodide (PI) staining using the Annexin V-fluorescein isothiocyanate (FITC) Apoptosis Detection kit (Beyotime Institute of Biotechnology, Haimen,
China). Following preconditioning as described, cells were lysed with lysis buffer (containing $10 \mathrm{mM}$ Tris, $10 \mathrm{mM}$ EDTA and $0.5 \%$ Triton $\mathrm{X}-100, \mathrm{pH} 7.5$ ). Then, cells were resuspended in binding buffer containing Annexin V-FITC and PI according to the manufacturer's instructions. All specimens were analyzed using a FACScan flow cytometer (BD Biosciences) and BD FACSDiva 6.1.3 software (BD Biosciences).

Sphere formation assays. To determine the self-renewal ability of GCSCs, sphere formation assays were performed. Briefly, cells were cultured in ultra-low attachment 24-well plates (Corning Life Sciences, Corning, NY, USA). Following transfection under the indicated conditions, cells were incubated with serum-free RPMI 1640 medium (Biosera, Nuaille, France) containing $20 \mathrm{ng} / \mathrm{ml}$ EGF and $10 \mathrm{ng} / \mathrm{ml} \mathrm{bFGF}$. After 3 weeks, the number of spheroid colonies were counted under a light microscope (CKX31; Olympus Corporation).

Cell invasion assays. Cell invasion assays were performed using 24-well Transwell chambers (8- $\mu \mathrm{m}$ pore polycarbonate membrane; BD Bioscience). Briefly, cells transfected with miR-483-5p mimics or miR-con were cultured in serum-free RPMI 1640 medium. Subsequently, the treated cells were seeded on the upper side of the membrane pre-coated with diluted Matrigel (BD Biosciences) in PBS. Then, medium containing $10 \%$ FBS was added into the lower chambers as a chemoattractant. After $48 \mathrm{~h}$ incubation, the non-invading cells inside the upper chamber were removed with a cotton swab. Cells that had migrated through the membrane to the lower surface were fixed with $4 \%$ paraformaldehyde, and then stained with $0.1 \%$ crystal violet (Shanghai Shenggong Biology Engineering Technology Service, Ltd., Shanghai, China). Quantification was performed by counting the number of cells in six high-powered fields in the center of each well under the CKX31 light microscope.

Western blotting. Cells were lysed with radioimmunoprecipitation assay lysis buffer (Beyotime Institute of Biotechnology) and the protein concentration was detected using a bicinchoninic acid protein assay kit (Beyotime Institute of Biotechnology). Following electrophoresis using sodium dodecyl sulfate-polyacrylamide gel electrophoresis with $12 \%$ polyacrylamide gel, the targeted proteins were electroblotted onto a polyvinylidene difluoride membrane (Merck Millipore, Darmstadt, Germany). Following incubation with 5\% non-fat milk to block the nonspecific binding, the membranes were immunoblotted with primary antibodies against $\beta$-catenin (1:5,000), cyclin D1 (1:10,000), Bcl-2 (1:1,000), Ki67 (1:1,000) and MMP-2 (1:1,000). After three washes with Tris-buffered saline with $0.05 \%$ Tween 20 (Sigma-Aldrich) at room temperature, each blot was incubated with goat anti-rabbit (1:1,000; cat. no. ab6789) and goat anti-mouse (1:5,000; cat. no. ab97051) horseradish peroxidase-conjugated secondary antibodies (Abcam) for $1 \mathrm{~h}$. Signals were detected with enhanced chemiluminescent detection reagent (Beyotime Institute of Biotechnology). $\beta$-actin served as loading control. The band intensity was scanned with the Gel Imaging System (UVP Company, Upland, CA, USA) and quantified using ImageJ 1.32 software (National Institutes of Health, Bethesda, MD, USA). All experiments were performed at least three times. 
A

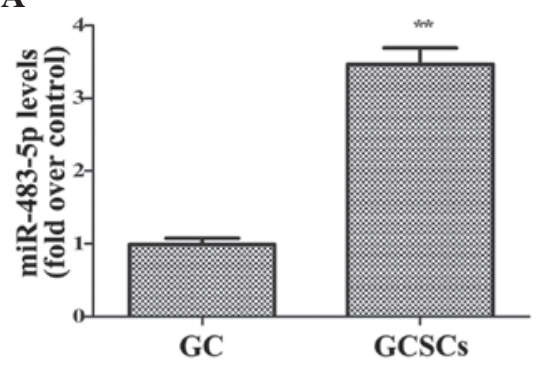

D

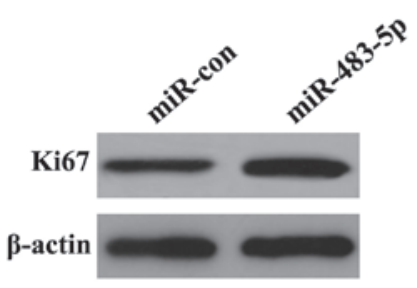

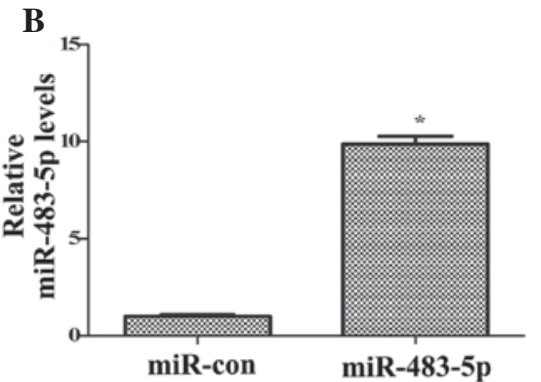

$\mathbf{E}$

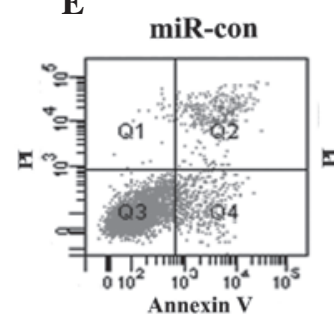

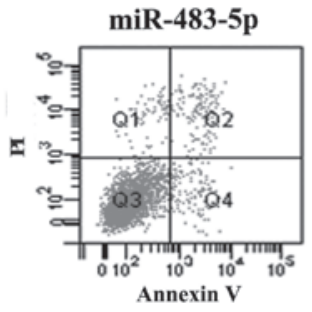

C
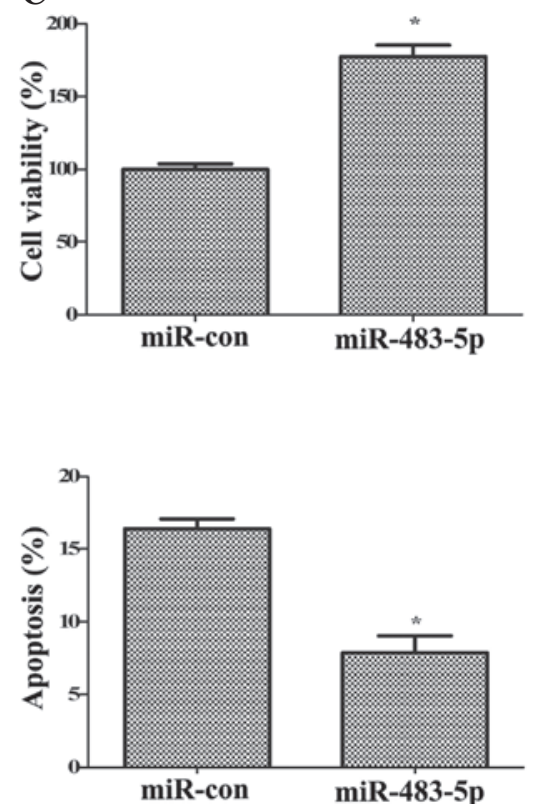

Figure 2. Overexpression of miR-483-5p promotes the growth of GCSCs. (A) The expression levels of miR-483-5p in the isolated GCSCs and gastric cancer cells $(\mathrm{MKN}-45){ }^{* *} \mathrm{P}<0.01$ vs. GC. (B) Following transfection with miR-483-5p mimics, the expression level of miR-483-5p was evaluated by reverse transcription-quantitative polymerase chain reaction. (C) The effect of miR-483-5p overexpression on cell proliferation was assessed by 3-(4,5-dimethylthiazol-2-yl)-2,5-diphenyltetrazolium bromide analysis. (D) The corresponding expression of marker molecule Ki67 was analyzed by western blotting. (E) The apoptotic rate of GCSCs was evaluated by staining with Annexin V/PI following miR-483-5p upregulation. *P<0.05 vs. miR-con. Values are presented as the mean \pm standard deviation. miR, microRNA; GC, gastric cancer; GCSCs, gastric cancer stem cells; PI, propidium iodide.

A

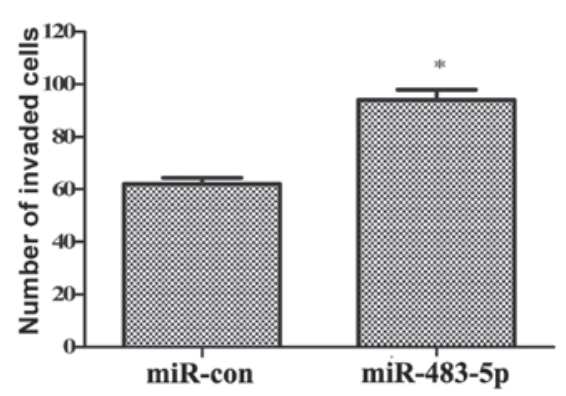

B

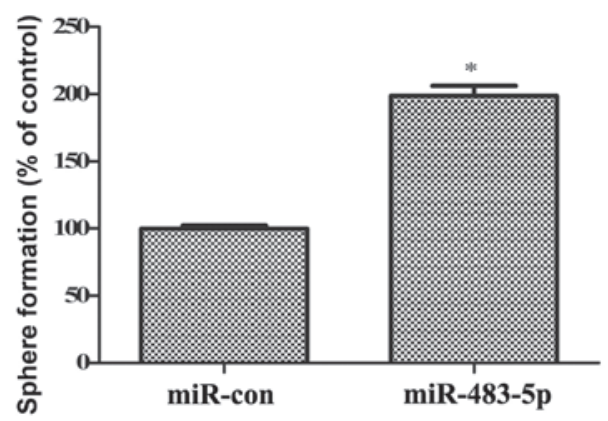

Figure 3. miR-483-5p promotes the invasion and self-renewal ability of GCSCs. (A) Cells were transfected with miR-483-5p mimics or miR-con, the corresponding cell invasive ability was evaluated by Transwell analysis. (B) The self-renewal ability of GCSCs was further confirmed by sphere formation assays. ${ }^{*} \mathrm{P}<0.05$ vs. miR-con. Values are presented as the mean \pm standard deviation. GCSCs, gastric cancer stem cells; miR, microRNA.

Statistical analysis. All results are representative of at least three experiments. Analysis was conducted using SPSS software (version 16.0; SPSS, Inc., Chicago, IL, USA). All data are presented as the mean \pm standard deviation. Significant differences between groups was analyzed using Student's t-test. $\mathrm{P}<0.05$ was considered to indicate a statistically significant difference.

\section{Results}

Identification of patient-derived GCSCs. FACS-sorted $\mathrm{CD} 44^{+} \mathrm{MKN}-45$ cells cultured in serum-free medium formed non-adherent, three-dimensional spheroid clusters, termed spheroid bodies. Notably, compared with parental cells, the spheroid bodies exhibited significantly increased self-renewal potential $(\mathrm{P}<0.05)$ detected by tumorspheric generation
(Fig. 1A). Additionally, the mRNA levels of CD44, Oct4, Nanog and Sox2, specific markers of GCSCs, were significantly increased in isolated spheroid body cells compared with parental cells ( $\mathrm{P}<0.05$; Fig. 1B). Furthermore, following further attachment and incubation in serum-containing medium, the spheroid body cells developed into elongated cells, and the mRNA levels of cytokeratin 14 (CK14) and CK18, markers of cell differentiation, were significantly upregulated compared with parental cells $(\mathrm{P}<0.05$; Fig. 1C). Thus, these results confirmed that isolated spheroid body cells exhibited increased self-renewal and differentiation potential, indicating the successful isolation of GCSCs from MKN-45 cells.

Level of miR-483-5p is elevated in GCSCs. Numerous previous studies have suggested that miR-483-5p expression is increased in several carcinomas and exerts an important 
A
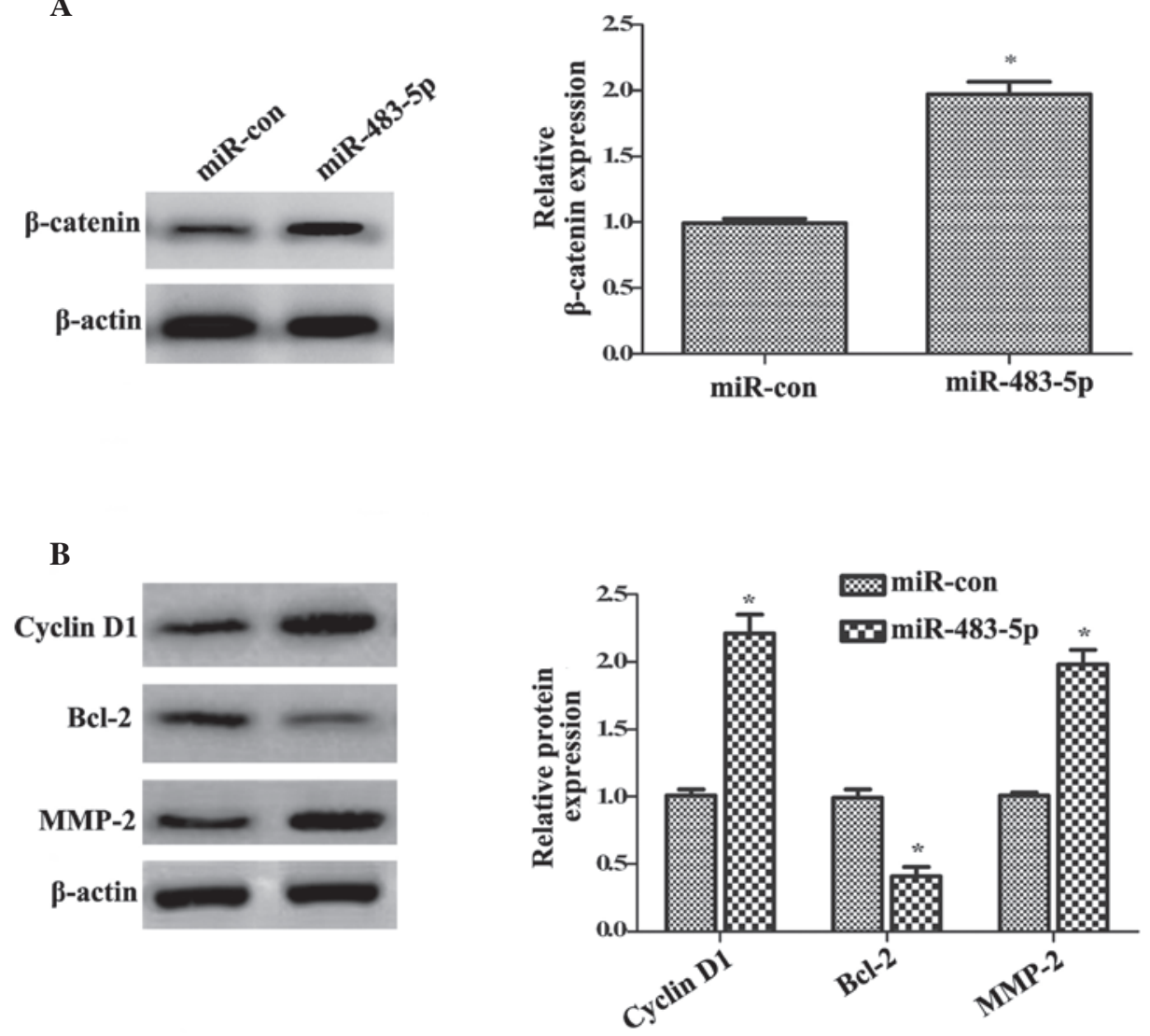

Figure 4. Effect of miR-483-5p on the activation of Wnt/ $/$-catenin signaling. Following overexpression of miR-483-5p in GCSCs, the expression levels of (A) $\beta$-catenin and (B) downstream targets (cyclin D1, Bcl-2 and MMP-2) were analyzed by western blotting. The corresponding quantitative analysis was performed using by Image $\mathrm{J}$ software. The corresponding effect on the expression of downstream targets were also evaluated. ${ }^{*} \mathrm{P}<0.05 \mathrm{vs}$. miR-con. Values are presented as the mean \pm standard deviation. GCSCs, gastric cancer stem cells; miR, microRNA; MMP-2; matrix metalloproteinase-2.

function in the development of cancer $(19,23)$. However, the importance of miR-483-5p in GC remains unclear. The present study explored the expression of miR-483-5p in GCSCs. As demonstrated in Fig. 2A, the expression levels of miR-483-5p were significantly upregulated in GCSCs compared with GC cells $(\mathrm{P}<0.01)$, indicating that miR-483-5p is important in the development of GC.

miR-483-5p overexpression promotes GCSC growth. To investigate the function of miR-483-5p in the tumorigenesis of GC, the effect of miR-483-5p on GCSC growth was analyzed. The current study overexpressed miR-483-5p in GCSCs by transfection with miR-483-5p mimics and measured the level with RT-qPCR (Fig. 2B). Functional analysis indicated that compared with control miR, miR-483-5p upregulation induced a 1.77 -fold increase in cell viability $(\mathrm{P}<0.05$; Fig. $2 \mathrm{C})$ and increased the expression of Ki67 (Fig. 2D), a common marker of cell proliferation. Apoptosis analysis demonstrated that the apoptotic rate was significantly reduced from 16.4 to $7.86 \%$ in control $\mathrm{miR}$ and $\mathrm{miR}-483-5 \mathrm{p}$ transfected cells, respectively $(\mathrm{P}<0.05$; Fig. $2 \mathrm{E})$. Together, these data indicated that miR-483-5p upregulation enhances GCSC growth by promoting cell proliferation and attenuating cell apoptosis.

Overexpression of miR-483-5p induces GCSC invasion and self-renewal. Based on the results of the current study, the effects of miR-283-5p on GCSC invasion and self-renewal were further investigated. As demonstrated in Fig. 3A, ectopic transfection of miR-483-5p significantly increased the invasiveness of GCSCs $(\mathrm{P}<0.05)$. The number of GCSCs that invaded the membrane was increased from 62 to 94 . The self-renewal ability of GCSCs, one of the most important characteristics of CSCs, was analyzed by sphere formation assays. Consistently, miR-483-5p overexpression induced a 1.98-fold increase in the number of spheres formed ( $\mathrm{P}<0.05$; Fig. $3 \mathrm{~B}$ ). These results corroborated that upregulation of miR-483-5p promotes the invasion and self-renewal of GCSCs.

miR-483-5p induces the activation of Wnt/ $\beta$-catenin pathway. Numerous studies have demonstrated that the abnormally high activation of the $\mathrm{Wnt} / \beta$-catenin pathway is pivotal for the initiation and development of various types of carcinoma $(24,25)$. To understand the underlying mechanism involved in the effect of miR-483-5p on GCSC growth, invasion and self-renewal, the Wnt/ $\beta$-catenin pathway was investigated. Western blotting demonstrated that transfection with miR-483-5p mimics significantly upregulated the $\beta$-catenin protein expression levels compared with control miR transfection ( 1.97-fold increase; $\mathrm{P}<0.05$; Fig. 4A). Furthermore, the significant upregulation of cyclin D1 and Bcl-2 protein levels was demonstrated in miR-483-5p-overexpressing cells compared with miR control cells $(\mathrm{P}<0.05$; Fig. 4B), which are key targeted molecules in the 
A

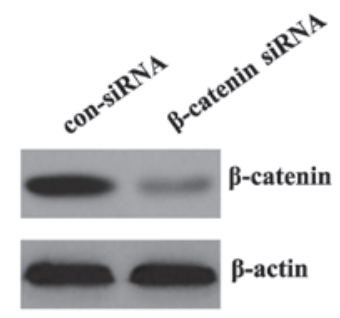

C

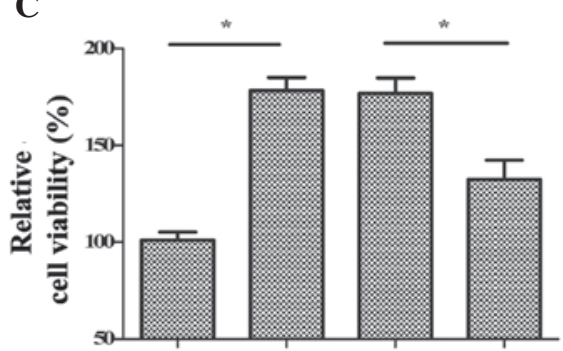

E

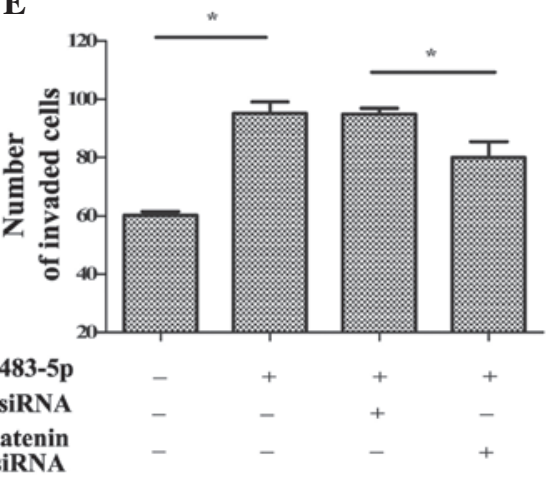

B

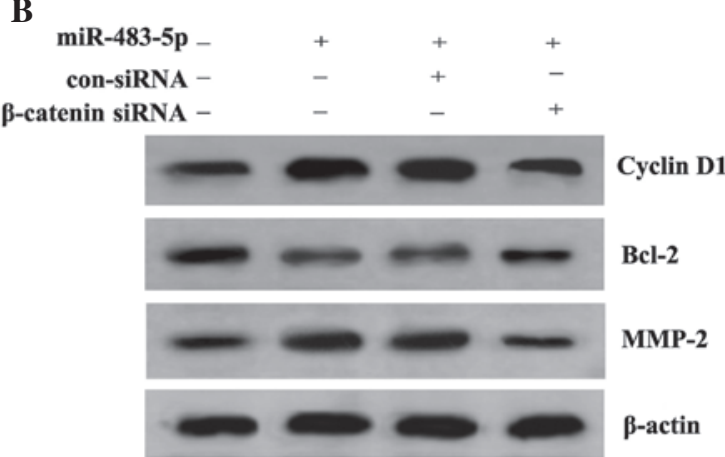

D

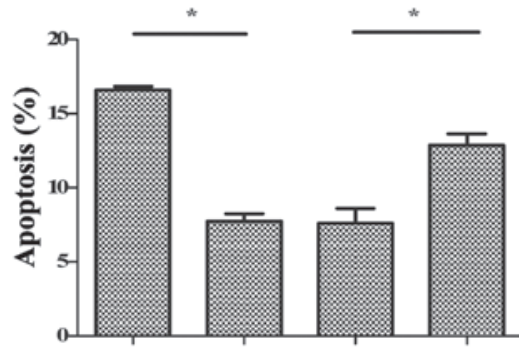

$\mathbf{F}$

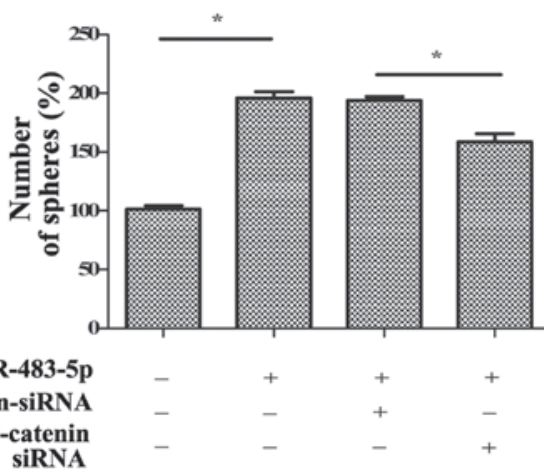

Figure 5. Wnt/ $\beta$-catenin pathway mediates miR-483-5p-induced GCSC growth, invasion and self-renewal. GCSCs were pretreated with $\beta$-catenin siRNA prior to miR-483-5p mimic transfection. (A) The silencing effect on $\beta$-catenin expression was determined by western blotting. (B) The effect of $\beta$-catenin silencing on miR-483-5p-induced activation of the Wnt/ $\beta$-catenin pathway was validated by detecting the expression of its downstream targets. (C) MTT assay was performed to demonstrated the effect of $\beta$-catenin siRNA transfection on miR-483-5p-induced increase in cell proliferation. The corresponding effect on (D) cell apoptosis, (E) invasion and (F) self-renewal were also measured. ${ }^{*} \mathrm{P}<0.05$. GCSC, gastric cancer stem cell; siRNA, small interfering RNA; miR, microRNA; MMP-2 matrix metalloproteinase-2.

Wnt/ $\beta$-catenin pathway and are involved in cell growth (25). Additionally, compared with miR control cells, the protein expression levels of MMP-2 were significantly increased following miR-483-5p overexpression. Taken together, the results of the present study suggested that miR-483-5p induces activation of the $\mathrm{Wnt} / \beta$-catenin pathway.

Wnt/ $\beta$-catenin signaling is involved in miR-483-5p-induced tumorigenesis. To elucidate the molecular mechanisms via which miR-483-5p affects the biological function of GCSCs, a specific $\beta$-catenin siRNA was synthesized. Following transfection with $\beta$-catenin siRNA, the expression levels of $\beta$-catenin were markedly reduced in GCSCs compared with control siRNA transfection (Fig. 5A). Additionally, the miR-483-5p-induced increase in the downstream targets of cyclin D1, Bcl-2 and MMP-2 were also markedly decreased compared with miR-483-5p and control siRNA transfected cells (Fig. 5B). Functional assays demonstrated that $\beta$-catenin silencing significantly reduced cell viability compared with
miR-483-5p transfection ( $\mathrm{P}<0.05$; Fig. 5C). Additionally, compared with miR-483-5p and control siRNA transfected cells, the inhibitory effect of miR-483-5p on cell apoptosis was ameliorated by $\beta$-catenin siRNA $(\mathrm{P}<0.05$; Fig. 5D). Furthermore, the increased cell invasion (Fig. 5E) and self-renewal (Fig. 5F) induced by miR-483-5p upregulation were significantly ablated following $\beta$-catenin siRNA transfection compared with miR-483-5p and control siRNA transfected cells $(\mathrm{P}<0.05)$. Therefore, the data of the current study indicated that miR-483-5p may promote GCSC growth, invasion and self-renewal via the $\mathrm{Wnt} / \beta$-catenin pathway.

\section{Discussion}

GC ranks as the leading cause of tumor-related mortality worldwide with a 5-year survival rate of only $28 \%$ (1). Despite the advances made in modern medicine, the incidence of GC remains high and constitutes a major health problem in developing countries with $\sim 700,000$ fatalities due to GC in 
2012. CSCs have become an important field in cancer research as their characteristic stemness properties may provide novel strategies for the treatment of patients with cancer $(4,6)$. The current study demonstrates the important finding that miR-483-5p levels were significantly upregulated in GCSCs. Notably, increased miR-483-5p promoted GCSC growth, invasion and self-renewal via the Wnt- $\beta$-catenin pathway. Therefore, the present study may clarify the importance of miR-483-5p in the development of GC.

miRNA dysregulation has previously been demonstrated to be involved in the development and progression of practically all types of cancer (12). Emerging evidence has confirmed an association between miRNAs and the pathological process of CSCs $(13,26)$. CSCs have been identified in various tumors, including GC, and exhibit mesenchymal and progenitor cell properties, including self-renewal and proliferative capacity $(27,28)$. Thus, CSCs may be associated with causing tumor initiation, metastasis and recurrence, and may be a potential target for the treatment of cancer. In the present study, the non-adherent spheroid body-forming MKN-45 cells cultured in stem cell conditioned medium exhibited increased self-renewal, levels of GCSC-associated markers (CD44, Oct4, Nanog and Sox2) and cell differentiation, indicating that the isolated cells possessed GCSC properties. Further analysis demonstrated increased expression of miR-483-5p in isolated GCSCs compared with GC cells, suggesting that miR483-5p is important for the development of GCSCs.

miR-483-5p was previously demonstrated to be overexpressed in several types of cancer, including multiple myeloma, hepatocellular carcinoma and adrenocortical cancer $(17,19)$. Furthermore, circulating miR-483-5p has previously been confirmed as a novel biomarker for the diagnosis and survival prediction of patients with cancer, indicating a pivotal function of miR-483-5p in the progression of carcinoma $(18,29)$. However, the underlying mechanism remains undefined in GC. To investigate the function of miR-483-5p in the development of GC, its effects on GCSC function were analyzed. Upregulation of miR-483-5p expression in GCSCs resulted in increased cell viability and reduced cell apoptosis, suggesting that miR-483-5p promotes GCSC growth. Further analysis demonstrated that miR-483-5p overexpression enhanced GCSC invasion. Notably, miR-483-5p overexpression increased the number of spheroid bodies formed, indicating that $\mathrm{miR}-483-5 \mathrm{p}$ is a positive regulator of GCSC self-renewal ability, which is one of the most important characteristics of CSCs. Thus, the data of the present study suggest that miR-483-5p may act as an oncogene during GC carcinogenesis by regulating GCSC function.

The canonical Wnt/ $\beta$-catenin signaling pathway has previously been demonstrated to be a major tumorigenesis pathway in various types of carcinoma $(23,30)$. Convincing evidence indicates that $\mathrm{Wnt} / \beta$-catenin signaling mediates tumor initiation and progression by regulating cell proliferation, invasion and metastasis. Emerging research has demonstrated the importance of $\mathrm{Wnt} / \beta$-catenin signaling in tumor stem and progenitor cells (31). The abnormal activation of the Wnt $/ \beta$-catenin pathway has previously been detected in CSCs from various types of carcinoma, including GC $(32,33)$. When Wnt/ $\beta$-catenin signaling was suppressed by chromobox 7, CSC proliferation, self-renewal and tumor initiating ability was shown to be inhibited in breast cancer (34). To further clarify the underlying mechanism associated with the effects of miR-483-5p on GCSC growth, invasion and self-renewal, the Wnt/ $\beta$-catenin signaling was investigated. In accordance with the hypothesis of the present study, miR-483-5p upregulation increased the protein expression levels of $\beta$-catenin, and upregulated cyclin D1, Bcl-2 and MMP-2 protein expression, which are common downstream molecules of Wnt/ $\beta$-catenin signaling involved in cell proliferation and invasion. Further functional assays demonstrated that inhibiting the pathway by $\beta$-catenin siRNA transfection attenuated the increases in miR-483-5p-induced cell growth, invasion and self-renewal ability. Thus, the data of the current study suggest that miR-483-5p may enhance GCSC function via activation of the Wnt/ $\beta$-catenin pathway, inducing cell growth, invasion and self-renewal.

In conclusion, the present study confirmed that miR-483-5p is increased in GCSCs derived from MKN-45 cells. Notably, miR-483-5p may be associated with GC maintenance by regulating growth, invasion and self-renewal via Wnt/ $\beta$-catenin signaling. Accordingly, the results of the current study may contribute to the understanding of how miR-483-5p regulates the development and pathological progression of GC, and provide a promising target for the treatment of GC.

\section{References}

1. Gomceli I, Demiriz B and Tez M: Gastric carcinogenesis. World J Gastroenterol 18: 5164-5170, 2012.

2. Siegel R, Naishadham D and Jemal A: Cancer statistics, 2012. CA Cancer J Clin 62: 10-29, 2012.

3. Brenner B, Hoshen MB, Purim O, David MB, Ashkenazi K, Marshak G, Kundel Y, Brenner R, Morgenstern S, Halpern M, et al: MicroRNAs as a potential prognostic factor in gastric cancer. World J Gastroenterol 17: 3976-3985, 2011.

4. Yu Z, Pestell TG, Lisanti MP and Pestell RG: Cancer stem cells. Int J Biochem Cell Biol 44: 2144-2151, 2012.

5. Xu G, Shen J, Ou Yang X, Sasahara M and Su X: Cancer stem cells: The 'heartbeat' of gastric cancer. J Gastroenterol 48: 781-797, 2013.

6. Visvader JE and Lindeman GJ: Cancer stem cells: Current status and evolving complexities. Cell Stem Cell 10: 717-728, 2012.

7. Conley SJ, Gheordunescu E, Kakarala P, Newman B, Korkaya H, Heath AN, Clouthier SG and Wicha MS: Antiangiogenic agents increase breast cancer stem cells via the generation of tumor hypoxia. Proc Natl Acad Sci USA 109: 2784-2789, 2012.

8. Yan K, Wu Q, Yan DH, Lee CH, Rahim N, Tritschler I, DeVecchio J, Kalady MF, Hjelmeland AB and Rich JN: Glioma cancer stem cells secrete Gremlin1 to promote their maintenance within the tumor hierarchy. Genes Dev 28: 1085-1100, 2014.

9. Visvader JE and Lindeman GJ: Cancer stem cells in solid tumours: Accumulating evidence and unresolved questions. Nat Rev Cancer 8: 755-768, 2008

10. Que T, Song Y, Liu Z, Zheng S, Long H, Li Z, Liu Y, Wang G, Liu Y, Zhou J, et al: Decreased miRNA-637 is an unfavorable prognosis marker and promotes glioma cell growth, migration and invasion via direct targeting Akt1. Oncogene 34: 4952-4963, 2015.

11. McGirt LY, Adams CM, Baerenwald DA, Zwerner JP, Zic JA and Eischen CM: miR-223 regulates cell growth and targets proto-oncogenes in mycosis fungoides/cutaneous T-cell lymphoma. J Invest Dermatol 134: 1101-1107, 2014.

12. Chen PS, Su JL and Hung MC: Dysregulation of microRNAs in cancer. J Biomed Sci 19: 90, 2012.

13. Croce CM and Calin GA: miRNAs, cancer, and stem cell division. Cell 122: 6-7, 2005.

14. Okada N, Lin CP, Ribeiro MC, Biton A, Lai G, He X, Bu P, Vogel H, Jablons DM, Keller AC, et al: A positive feedback between $\mathrm{p} 53$ and miR-34 miRNAs mediates tumor suppression. Genes Dev 28: 438-450, 2014.

15. Bao B, Wang Z, Ali S, Ahmad A, Azmi AS, Sarkar SH, Banerjee S, Kong D, Li Y, Thakur S and Sarkar FH: Metformin inhibits cell proliferation, migration and invasion by attenuating CSC function mediated by deregulating miRNAs in pancreatic cancer cells. Cancer Prev Res (Phila) 5: 355-364, 2012. 
16. Liu C, Kelnar K, Vlassov AV, Brown D, Wang J and Tang DG: Distinct microRNA expression profiles in prostate cancer stem/progenitor cells and tumor-suppressive functions of let-7. Cancer Res 72: 3393-3404, 2012.

17. Chabre O, Libé R, Assié G, Barreau O, Bertherat J, Bertagna X, Feige JJ and Cherradi N: Serum miR-483-5p and miR-195 are predictive of recurrence risk in adrenocortical cancer patients. Endocr Relat Cancer 20: 579-594, 2013.

18. Zhang Z, Ge S, Wang X, Yuan Q, Yan Q, Ye H, Che Y, Lin Y, Zhang J and Liu P: Serum miR-483-5p as a potential biomarker to detect hepatocellular carcinoma. Hepatol Int 7: 199-207, 2013.

19. Song Q, Xu Y, Yang C, Chen Z, Jia C, Chen J, Zhang Y, Lai P, Fan $\mathrm{X}$, Zhou $\mathrm{X}$, et al: miR-483-5p promotes invasion and metastasis of lung adenocarcinoma by targeting RhoGDI1 and ALCAM. Cancer Res 74: 3031-3042, 2014.

20. Liu J, Ma L, Xu J, Liu C, Zhang J, Liu J, Chen R and Zhou Y: Spheroid body-forming cells in the human gastric cancer cell line MKN-45 possess cancer stem cell properties. Int J Oncol 42: 453-459, 2013.

21. Wang L, Shi M, Hou S, Ding B, Liu L, Ji X, Zhang J and Deng Y: MiR-483-5p suppresses the proliferation of glioma cells via directly targeting ERK1. FEBS Lett 586: 1312-1317, 2012.

22. Livak KJ and Schmittgen TD: Analysis of relative gene expression data using real-timequantitative PCR and the 2(-Delta Delta C(T)) Method. Methods 25: 402-408, 2001.

23. Wu H, Echt CS, Popp MP and Davis JM: Molecular cloning, structure and expression of an elicitor-inducible chitinase gene from pine trees. Plant Mol Biol 33: 979-987, 1997.

24. White BD, Chien AJ and Dawson DW: Dysregulation of Wnt/ $\beta$-catenin signaling in gastrointestinal cancers. Gastroenterology 142: 219-232, 2012.
25. Yu T, Liu K, Wu Y, Fan J, Chen J, Li C, Yang Q and Wang Z: MicroRNA-9 inhibits the proliferation of oral squamous cell carcinoma cells by suppressing expression of CXCR4 via the Wnt/ $\beta$-catenin signaling pathway. Oncogene 33: 5017-5027, 2014

26. Brower JV, Clark PA, Lyon W and Kuo JS: MicroRNAs in cancer: Glioblastoma and glioblastoma cancer stem cells. Neurochem Int 77: 68-77, 2014.

27. Jiang J, Zhang Y, Chuai S, Wang Z, Zheng D, Xu F, Li C, Liang Y and Chen Z: Trastuzumab (herceptin) targets gastric cancer stem cells characterized by CD90 phenotype. Oncogene 31: 671-682, 2012.

28. Templeton AK, Miyamoto S, Babu A, Munshi A and Ramesh R: Cancer stem cells: Progress and challenges in lung cancer. Stem Cell Investigation 1: 9, 2014.

29. Qu X, Zhao M, Wu S, Yu W, Xu J, Xu J, Li J and Chen L: Circulating microRNA 483-5p as a novel biomarker for diagnosis survival prediction in multiple myeloma. Med Oncol 31: 219, 2014.

30. Arensman M, Lay AR, Kulikauskas RM, Chien AJ and Dawson DW: Wnt $/ \beta$-catenin transcriptional activation promotes tumorigenesis and predicts survival in pancreatic cancer. Cancer Res 73: 4011, 2013.

31. Holland JD, Klaus A, Garratt AN and Birchmeier W: Wnt signaling in stem and cancer stem cells. Curr Opin Cell Biol 25: 254-264, 2013.

32. Cai $\mathrm{C}$ and Zhu $\mathrm{X}$ : The $\mathrm{Wnt} / \beta$-catenin pathway regulates self-renewal of cancer stem-like cells in human gastric cancer. Mol Med Rep 5: 1191-1196, 2012.

33. Li J and Zhou BP: Activation of $\beta$-catenin and Akt pathways by Twist are critical for the maintenance of EMT associated cancer stem cell-like characters. BMC Cancer 11: 49, 2011.

34. Kim HY, Park JH, Won HY, Lee JY and Kong G: CBX7 inhibits breast tumorigenicity through DKK-1-mediated suppression of the Wnt/ $\beta$-catenin pathway. FASEB J 29: 300-313, 2015. 